\title{
Memory effects in the relaxation of a confined granular gas
}

\author{
J. Javier Brey, M. I. García de Soria, P. Maynar, and V. Buzón \\ Física Teórica, Universidad de Sevilla, Apartado de Correos 1065, E-41080, Sevilla, Spain \\ (Received 25 April 2014; revised manuscript received 28 July 2014; published 12 September 2014)
}

\begin{abstract}
The accuracy of a model to describe the horizontal dynamics of a confined quasi-two-dimensional system of inelastic hard spheres is discussed by comparing its predictions for the relaxation of the temperature in a homogenous system with molecular dynamics simulation results for the original system. A reasonably good agreement is found. Next the model is used to investigate the peculiarities of the nonlinear evolution of the temperature when the parameter controlling the energy injection is instantaneously changed while the system was relaxing. This can be considered as a nonequilibrium generalization of the Kovacs effect. It is shown that, in the low-density limit, the effect can be accurately described by using a simple kinetic theory based on the first Sonine approximation for the one-particle distribution function. Some possible experimental implications are indicated.
\end{abstract}

DOI: 10.1103/PhysRevE.90.032207

PACS number(s): 45.70.Mg, 05.20.Jj, 51.10.+y

\section{INTRODUCTION}

Granular gases [1] are composed of macroscopic particles, grains, which do not conserve the kinetic energy when they collide. As such, there is no equilibrium state, since an isolated undriven system will cool continuously. To reach and maintain a steady state, a permanent injection of energy is needed. Typically, this is done by injecting energy through the boundaries or by means of external fields acting on the bulk of the system. As a consequence, the system develops large inhomogeneities, often associated to the presence of instabilities. A driven homogeneous steady state can be obtained if the grains are submitted to a (fictitious) external noise force, usually referred to as a stochastic thermostat [2-4]. Nevertheless, the possible relationship of this kind of forces with real experimental situations has not been discussed in detail to the best of our knowledge.

In the past few years a quite interesting alternative, overcoming the above difficulty, has been addressed. The idea is to consider a granular gas confined to a quasitwo-dimensional geometry by placing it between two large parallel horizontal plates separated a distance smaller than two grain diameters, so the system is actually a granular monolayer [5,6]. To keep the system fluidized and render a steady state possible, it is mechanically vibrated in the vertical direction. The two-dimensional dynamics of the projection of the grains motion on an horizontal plane is considered. It has been found experimentally that the behavior of the projected system resembles that of a two-dimensional fluid. Moreover, it remains homogeneous over a wide range of values of the parameters controlling the experiment, eventually reaching a steady homogeneous state. On the other hand, when varying the average density and the intensity of the vibration, the system exhibits a series of phase transitions [5-8].

A common paradigm for granular gases, employed in many theories and simulations, is that of a collection of uniform smooth inelastic hard spheres or disks, whose collisions are characterized by a constant, velocity-independent, coefficient of normal restitution [9]. Although this model has several shortcomings, it enables us to make the relevance of inelasticity clear in the simplest possible scenario. Very recently [10], this model has been modified trying to mimic the horizontal dynamics of a vibrated fluid of inelastic hard spheres confined to a quasi-two-dimensional geometry, i.e., the kind of system described above. To do so, the inelastic collision rule for hard disks has been changed in order to account for the mechanism by which the kinetic energy given to the vertical motion of the particles by the vibrating walls is transferred to the horizontal degrees of freedom through the collisions between particles. Since the model is formulated in terms of a deterministic instantaneous collision rule, the methods of nonequilibrium statistical mechanics and kinetic theory developed for inelastic hard spheres and disks $[11,12]$ can be easily extended to it [13]. In this way, it has been shown that it admits a solution describing a homogeneous steady state, similarly to what happens in the experiments [13].

A first relevant question is to somehow evaluate the accuracy of the model to describe the original quasi-twodimensional vibrated system and, if possible, to compare it with the stochastic thermostat model mentioned at the beginning. Some information about this issue will be provided here by analyzing the relaxation of the temperature towards its steady value as predicted by both models and the results obtained by means of molecular dynamics (MD) simulations of the the original three-dimensional confined system.

As a direct implication of the existence of a mechanism of energy injection, there is homogeneous hydrodynamics in the collisional model proposed by Brito et al. [10], i.e., there is a time scale over which the time evolution of the (granular) temperature in a homogeneous system obeys a macroscopic differential equation whose solution only depends on the initial temperature [14]. In the context of kinetic theory, the existence of homogeneous hydrodynamics follows from the one-particle distribution function of the system being normal, meaning that all its time dependence occurs through the temperature $[15,16]$. Therefore, two homogeneous hydrodynamic states of a given system are the same state if both have the same temperature. The above is true not only at the macroscopic level but also when the system is described by means of kinetic theory or nonequilibrium statistical mechanics using its distribution function. Of course, there is the possibility of different homogeneous states having the same temperature, but only one of them, at the most, can be described by hydrodynamics. The above comments apply, in particular, to the steady state, 
since it is the hydrodynamic state reached by the system in the long time limit.

Consider a confined quasi-two-dimensional system which is in the homogeneous stationary state with a temperature $T_{1}$. Of course, this refers to the projected two-dimensional system. Then the vibration intensity is instantaneously decreased so the new stationary temperature $T_{2}$ is lower, and the system will cool towards it. At a later time $t_{0}$, the vibration intensity is again modified, now in such a way that the instantaneous value of the temperature $T\left(t_{0}\right)$ is equal to the steady temperature corresponding to the new vibration intensity. Although the temperature of the system has its long-time steady value, it is not expected to remain constant but to deviate from this steady value, going through a maximum or a minimum and, finally, tending to stationarity. The reason to expect this behavior is that, although the system has its steady temperature, it is not in the steady state. Even more, it cannot be in a hydrodynamic state for the reasons mentioned above. As a consequence, the macroscopic equation for the evolution of the temperature cannot be used to describe its evolution following the change of the vibration intensity at $t_{0}$. The experiment just described, as well as the predicted behavior of the system, can be considered as reminiscent of the Kovacs memory effect occurring in the relaxation of molecular systems towards equilibrium [17] that has been extensively studied in the last years [18-22]. Nevertheless, it is important to realize that in the above process we are dealing with an intrinsic nonequilibrium system trying to approach its steady state, while the original Kovacs effect refers to the relaxation towards equilibrium. Moreover, while Kovacs carried out an instantaneous quench of the system and studied the evolution of a macroscopic quantity, namely the volume of the system, here what is instantaneously changed is the external parameter controlling the energy injection, and it is the time evolution of the temperature what is followed.

One of the aims of this paper is to investigate the presence of a Kovacs-like effect in the collisional model for a confined granular gas. It will be shown that this is the case and that, for a low-density granular gas, the effect is well captured by kinetic theory at the level of the (inelastic) Boltzmann equation in the first Sonine approximation. Actually, the effect shows up when the characteristic speed involved in the formulation of the model is modified to increase or to decrease the value of the steady temperature and, therefore, leads to a heating and cooling process, respectively. A study of the same Kovacs-like effect in the thermostated model was carried out very recently in Ref. [23]. Since the method used there differs from the one developed here, a brief summary of how the latter can be applied to the thermostat model is presented Appendix B. The obtained results are consistent with those derived in Ref. [23]. The main features of the effect exhibited by both models will be compared in last section of the paper.

The remaining of the paper is organized as follows. In the next section, the stochastic thermostat model $[2,4]$ and the collisional model $[10,13]$ leading to homogeneous steady states of a system of inelastic hard disks are shortly reviewed. The macroscopic equations for the evolution of the granular temperature and the expressions for its steady value in both models are noted. By fitting the steady temperatures predicted by the models to the MD results obtained for the horizontal motion of a confined quasi-two-dimensional system of inelastic hard spheres under vibration, the phenomenological parameter appearing in each of the models is determined as a function of the vibration intensity. Then these values are used into the equations for the evolution of the temperature predicted by each model, and their solutions are compared with MD simulation results, without any new fitting parameter. The agreement of the collisional model is definitely better than that of the stochastic thermostat model. In Sec. III, the steady velocity distribution is considered. The MD results indicate that the shape of the distribution is very close to a Gaussian. Therefore, the first Sonine approximation has been considered, and the comparison of the two models with the simulation data has been carried out by using the fourth moment of the velocity distribution, which is closely related to the coefficient of the first Sonine correction term. Although both the stochastic and the collisional models accurately predict that the Sonine coefficient is very small, they fail to describe its qualitative dependence with the coefficient of normal restitution of the particles.

In Sec. IV, an ideal experiment in which the characteristic speed of the collisional model is suddenly changed is described and analyzed in the low-density limit by using kinetic theory. Its predictions for the nonlinear time evolution of the temperature after the jump are compared with numerical results obtained using the direct simulation Monte Carlo method. A reasonable good agreement is found. The last section contains some summarizing remarks as well as some possible experimental implications of the results reported in the paper. Finally, Appendix A contains some mathematical details, and in Appendix B, the method developed in the paper is applied to the stochastic thermostat model, and results consistent with those presented in Ref. [23] are found.

\section{TWO MODELS OF A VIBRATED CONFINED GRANULAR GAS}

The system considered is a vibrated granular gas confined to a quasi-two-dimensional geometry. The grains are smooth, inelastic hard spheres of mass $m$ and diameter $\sigma$. Collisions between particles are characterized by a constant, velocity-independent, coefficient of normal restitution $\alpha$, defined in the interval $0<\alpha \leqslant 1$. Therefore, when two particles, $i$ and $j$, collide their velocities are instantaneously modified according with

$$
\begin{gathered}
\boldsymbol{v}_{i} \rightarrow \boldsymbol{v}_{i}^{\prime}=\boldsymbol{v}_{i}-\frac{1+\alpha}{2} \boldsymbol{v}_{i j} \cdot \widehat{\boldsymbol{\sigma}} \widehat{\boldsymbol{\sigma}}, \\
\boldsymbol{v}_{j} \rightarrow \boldsymbol{v}_{j}^{\prime}=\boldsymbol{v}_{j}+\frac{1+\alpha}{2} \boldsymbol{v}_{i j} \cdot \widehat{\boldsymbol{\sigma}} \widehat{\boldsymbol{\sigma}},
\end{gathered}
$$

where $\boldsymbol{v}_{i j} \equiv \boldsymbol{v}_{i}-\boldsymbol{v}_{j}$ is the relative velocity prior collision and $\widehat{\sigma}$ is a unit vector directed from the center of particle $j$ to the center of particle $i$ at contact.

The system is confined by means of two parallel plates separated a distance $h, \sigma<h<2 \sigma$. The bottom plate is vibrating, and for the sake of simplicity, the limit of small amplitude and very high frequency will be supposed. Then the bottom wall moves in a sawtooth way, and every colliding particle finds it at a fixed position and moving with a constant speed $v_{b}$ directed upwards [24]. The upper wall is at rest. Collisions of the particles with both walls are elastic. As the 
particles collide with the bottom wall, energy is transferred to the vertical degree of freedom of the grains. Afterwards, this energy is transferred to the horizontal degrees of freedom of the grains in the collisions between them. The interest here is on the behavior of the system when observed from above and the two-dimensional dynamics in the horizontal plane is analyzed. No effect of gravity is considered. The system has been designed trying to mimic some experimental setups reported in the literature $[8,25]$. Moreover, a similar confined granular system, but without the top lid, has been investigated [26]. In both cases, one of the goals is to avoid the large inhomogeneities usually developed in the bulk of a granular gas when energy is injected inhomogeneously through the boundaries $[27,28]$. In the confined quasi-two-dimensional granular gas considered here, homogeneous steady states are possible because the energy injection occurs throughout the whole system [29-32].

Molecular dynamics (MD) simulations of the above system have been carried out by using the event-driven method [33,34]. Periodic boundary conditions have been used in the horizontal plane. In the initial state, grains were homogeneosuly distributed in the horizontal plane equidistant from both walls, and the initial velocities were assigned accordingly with a Gaussian distribution of zero average. Details of these initial configurations are always lost in a few collisions per particle and, if the two-dimensional dynamics is considered, the system reaches a time-dependent homogeneous state. In the long time limit, the system eventually remains in a homogeneous steady state, although when varying the solid fraction and the vibration amplitude, the system exhibits a solid-liquid transition, with different possible solid phases [29-31]. For fixed values of the geometrical parameters defining the particles and the system, the density, and the coefficient of normal restitution, the steady temperature depends on the velocity $v_{b}$ of the vibrating wall.

Two models proposed in the literature leading to a homogeneous steady state of a granular gas will be considered in the following. In the first one, the granular medium is subjected to a bulk injection of energy by means of a stochastic force [2]. The model was formulated without having any particular application in mind. On the other hand, the second model was designed as a collisional model trying to mimic the effective two-dimensional dynamics of the confined quasi-two-dimensional granular gas [10]. Next, both models will be briefly summarized, focusing on the subject of interest here, namely their predictions for the homogeneous macroscopic evolution of the two-dimensional granular gas. Let us mention that the stochastic model being considered here is not the only existing one. For instance, another model incorporating an additional friction term balancing the noise has been formulated to investigate hydrodynamical fluctuations in granular gases [35].

\section{A. The stochastic thermostat}

In the stochastic thermostat model, energy is uniformly supplied to the system by means of a white noise force acting independently on each grain [2,4,36-39], so the one-particle velocity distribution, $f(\boldsymbol{v}, t)$, of a dilute homogeneous gas of inelastic hard disks obeys the Boltzmann-Fokker-Planck equation,

$$
\begin{aligned}
\frac{\partial}{\partial t} f\left(\boldsymbol{v}_{1}, t\right)= & \sigma \int d \boldsymbol{v}_{2} \int d \widehat{\boldsymbol{\sigma}} \theta\left(\boldsymbol{v}_{12} \cdot \widehat{\boldsymbol{\sigma}}\right) \boldsymbol{v}_{12} \cdot \widehat{\boldsymbol{\sigma}} \\
& \times\left[\alpha^{-2} b_{\sigma}^{-1}(1,2)-1\right] f\left(\boldsymbol{v}_{1}, t\right) f\left(\boldsymbol{v}_{2}, t\right) \\
& +\frac{\xi_{0}^{2}}{2} \frac{\partial^{2}}{\partial \boldsymbol{v}_{1}^{2}} f\left(\boldsymbol{v}_{1}, t\right) .
\end{aligned}
$$

Here the operator $b_{\sigma}^{-1}(1,2)$ changes the velocities $\boldsymbol{v}_{1}$ and $\boldsymbol{v}_{2}$ to its right into the precollisonal values corresponding to the collision rule given in Eq. (1) and (2), i.e.,

$$
\begin{aligned}
& b_{\boldsymbol{\sigma}}^{-1}(1,2) \boldsymbol{v}_{1}=\boldsymbol{v}_{1}-\frac{1+\alpha}{2 \alpha} \boldsymbol{v}_{12} \cdot \widehat{\boldsymbol{\sigma}} \widehat{\boldsymbol{\sigma}}, \\
& b_{\boldsymbol{\sigma}}^{-1}(1,2) \boldsymbol{v}_{2}=\boldsymbol{v}_{2}+\frac{1+\alpha}{2 \alpha} \boldsymbol{v}_{12} \cdot \widehat{\boldsymbol{\sigma}} \widehat{\boldsymbol{\sigma}} .
\end{aligned}
$$

The quantity $\xi_{0}$ measures the magnitude of the fluctuating force and it must be considered as a parameter defining the model. Approximating the distribution function by a Gaussian, an evolution equation for the temperature $T(t)$ is obtained by taking moments in the above Boltzmann equation,

$$
\frac{\partial T}{\partial t}=-\Gamma+m \xi_{0}^{2}
$$

where

$$
\Gamma \equiv \frac{\left(1-\alpha^{2}\right) \omega T}{2}
$$

and

$$
\omega \equiv 2 \pi n \sigma\left(\frac{T}{\pi m}\right)^{2 / 3},
$$

$n$ being the number density of disks. Therefore, the steady temperature $T_{\mathrm{st}}$ reached by the system in the long time limit is given by

$$
T_{\mathrm{st}}=m\left[\frac{\xi_{0}^{2}}{\pi^{1 / 2}\left(1-\alpha^{2}\right) n \sigma}\right]^{2 / 3}
$$

This expression has been used to determine the unknown parameter $\xi_{0}$ of the model as a function of the velocity $v_{b}$ of the vibrating wall in the original quasi-two-dimensional system. The steady temperature of the effective two-dimensional gas in the latter has been measured by means of MD simulations as a function of $v_{b}$. Equation (9) provides the value predicted by the stochastic model as a function of the noise amplitud $\xi_{0}$. It is then trivial to fit $\xi_{0}$ as a function of $v_{b}$, for fixed values of all the other parameters, so Eq. (9) yields the dependence of $T_{\mathrm{st}}$ on $v_{b}$ measured in the MD simulations. In Fig. 1, the results obtained for a system of $N=500$ inelastic hard spheres with a coefficient of normal restitution $\alpha=0.8$ are shown. The unit cell is a parallelepipedal of basis $L \times L$, with $L=129 \sigma$ and height $h=1.5 \sigma$. Then the three-dimensional number density is $n=0.02 \sigma^{-3}$, which corresponds to a dilute granular gas. The two-dimensional number density entered into Eq. (9) has been computed by considering $N$ disks of diameter $\sigma$ inside the square of size $L \times L$. 


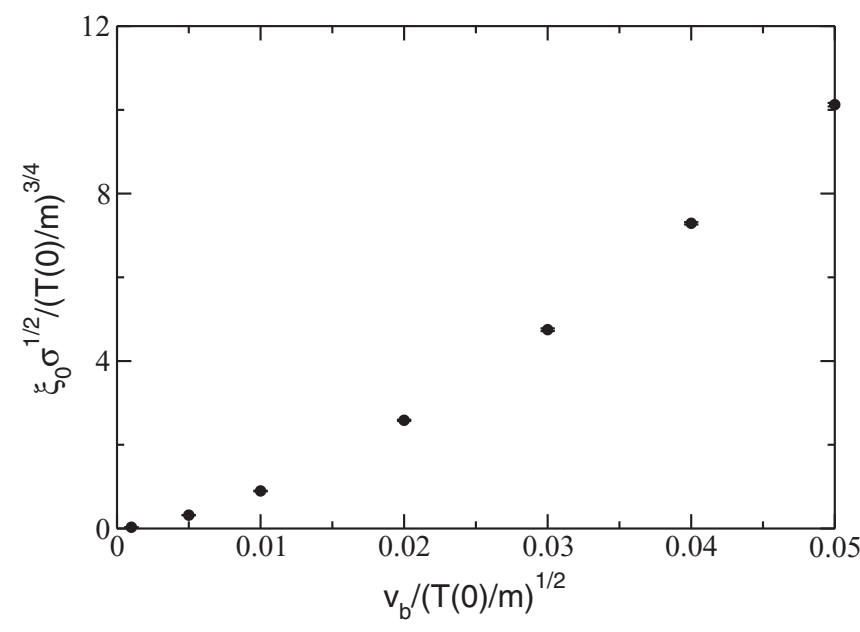

FIG. 1. Noise strength $\xi_{0}$ of the stochastic thermostat model for the two-dimensional dynamics as a function of the velocity $v_{b}$ of the vibrating wall in the three-dimensional system, obtained in the way discussed in the main text. Dimensionless quantities have been defined as indicated in the respective labels, with $T(0)$ being the initial temperature of the (three-dimensional) gas. The coefficient of normal restitution is $\alpha=0.8$ and the density of the gas $n=0.02 \sigma^{-3}$.

\section{B. The collisional model}

The idea in the collisional model is to replace the actual three-dimensional collision rule, Eqs. (1) and (2), by an effective two-dimensional one in order to describe the dynamics in the horizontal plane [10]. Then a system of hard disks is considered, in which collisions change the velocities according to

$$
\begin{gathered}
\boldsymbol{v}_{i} \rightarrow \boldsymbol{v}_{i}^{\prime}=\boldsymbol{v}_{i}-\frac{1+\alpha}{2} \boldsymbol{v}_{i j} \cdot \widehat{\boldsymbol{\sigma}} \widehat{\boldsymbol{\sigma}}+\Delta \widehat{\boldsymbol{\sigma}}, \\
\boldsymbol{v}_{j} \rightarrow \boldsymbol{v}_{j}^{\prime}=\boldsymbol{v}_{j}+\frac{1+\alpha}{2} \boldsymbol{v}_{i j} \cdot \widehat{\boldsymbol{\sigma}} \widehat{\boldsymbol{\sigma}}-\Delta \widehat{\boldsymbol{\sigma}} .
\end{gathered}
$$

Here $\Delta$ is some positive characteristic speed. It tries to describe in an effective way the mechanism for which energy is injected into the horizontal degrees of freedom of the inelastic hard spheres.

In the low-density limit, the dynamics of the system can be accurately described by an inlelastic Boltzmann equation [13]. From it, an evolution equation for the temperature in homogeneous situations can be derived. It reads as follows [14]:

$$
\frac{\partial T(t)}{\partial t}=-\zeta_{H}(t) T(t)
$$

with the homogeneous rate of change of the temperature $\zeta_{H}(t)$ given by

$$
\begin{aligned}
\zeta_{H}(t)= & (2 \pi)^{1 / 2} n \sigma v_{0}(t)\left[\frac{1-\alpha^{2}}{2}\left(1+\frac{3 a_{2}}{16}\right)\right. \\
& \left.-\alpha\left(\frac{\pi}{2}\right)^{1 / 2} \Delta^{*}-\left(1-\frac{a_{2}}{16}\right) \Delta^{* 2}\right],
\end{aligned}
$$

where $v_{0}(t) \equiv(2 T(t) / m)^{1 / 2}$ is a thermal velocity and $\Delta^{*} \equiv$ $\Delta / v_{0}(t)$. Upon deriving the above expression, the first Sonine approximation [15] for the one-particle distribution function has been assumed,

$$
f(\boldsymbol{v}, t) \approx n v_{0}(t)^{-2} \pi^{-1} e^{-c^{2}}\left[1+a_{2}(t) S^{(2)}\left(c^{2}\right)\right] .
$$

In this expression,

$$
\boldsymbol{c} \equiv \frac{\boldsymbol{v}}{v_{0}(t)}
$$

and

$$
S^{(2)}\left(c^{2}\right) \equiv 1-2 c^{2}+\frac{c^{4}}{2} .
$$

It is worth noting that Eqs. (12) and (13) hold as long as the distribution function can be approximated by (14), without requiring any additional hypothesis. The expression of the Sonine coefficient $a_{2}(t)$ in the hydrodynamic regime has been determined in Ref. [14] and it will be considered in Sec. IV. For the time being, an accurate-enough result for the steady temperature is obtained by using the Gaussian approximation for the distribution function, i.e., by putting $a_{2}=0$ in Eq. (13). In this case, the steady temperature predicted by the collisional model is $[10,13]$

$$
T_{\mathrm{st}}=\frac{\pi m \alpha^{2}}{4\left(1-\alpha^{2}\right)^{2}}\left[1+\sqrt{1+\frac{4\left(1-\alpha^{2}\right)}{\pi \alpha^{2}}}\right]^{2} \Delta^{2} .
$$

Now the same procedure as for the stochastic thermostat model can be used to determine the value of the characteristic speed $\Delta$ from the MD simulation results for the present model. In Fig. 2, the characteristic speed $\Delta$ is plotted as a function of the velocity of the vibrating wall $v_{b}$ for the same system as considered in Fig. 1. A quite perfect linear relationship is observed, consistently with dimensional analysis. It is important to realize a conceptually relevant difference between the stochastic and the collisional models. While the latter predicts a stationary temperature that is independent from the

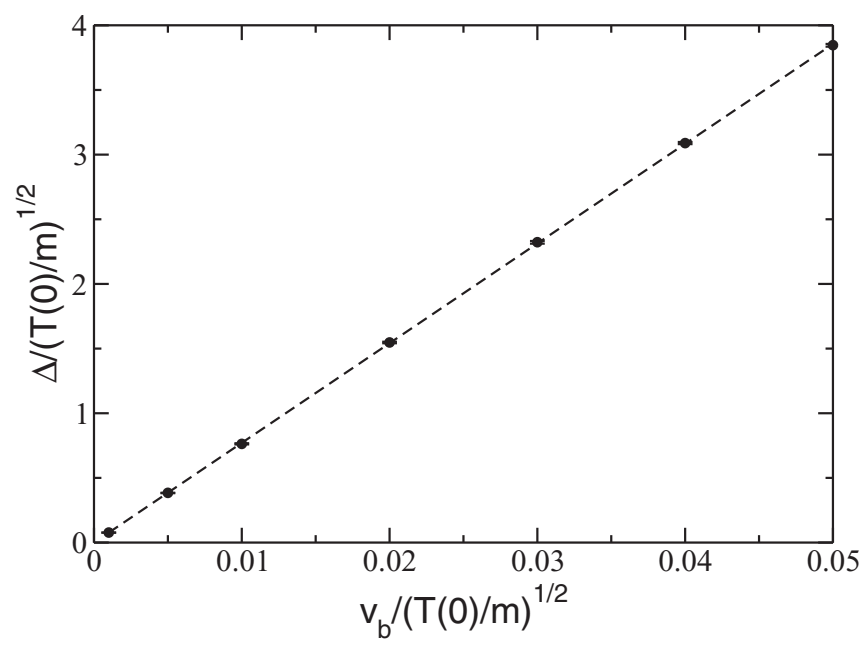

FIG. 2. Characteristic speed $\Delta$ of the collisional model for the two-dimensional dynamics as a function of the velocity $v_{b}$ of the vibrating wall in the three-dimensional system, obtained in the way discussed in the main text. Dimensionless quantities have been defined as indicated in the respective labels, with $T(0)$ being the initial temperature of the (three-dimensional) gas. The coefficient of normal restitution is $\alpha=0.8$ and the density of the gas $n=0.02 \sigma^{-3}$. The straight dashed line is just a guide for the eye. 


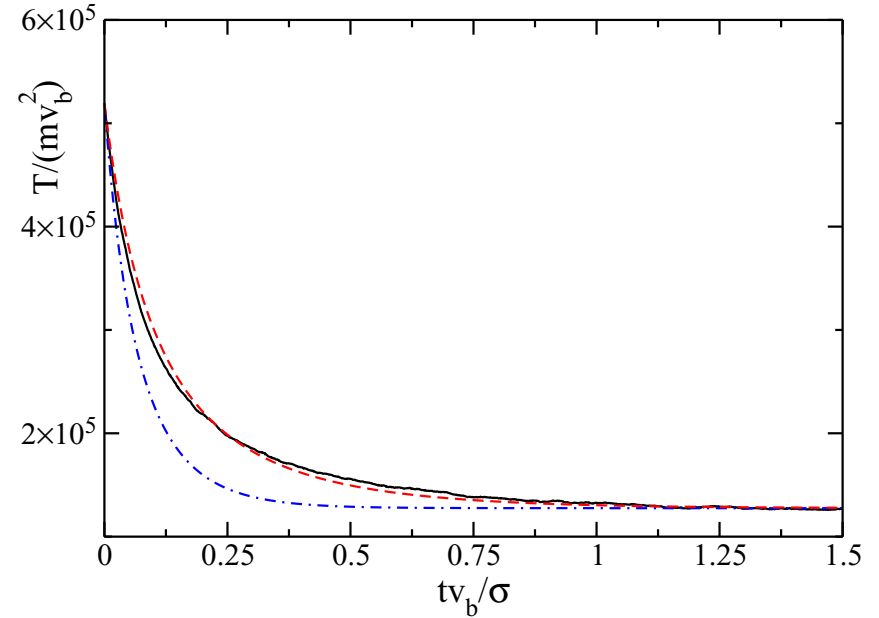

FIG. 3. (Color online) Relaxation of the temperature in a system with a coefficient of normal restitution $\alpha=0.8$, height $h=1.5 \sigma$, and three-dimensional density $n=0.02 \sigma^{-3}$. The velocity of the vibrating wall is $v_{b}=10^{-3}(T(0) / m)^{1 / 2}$. Both temperature and time are measured in the dimensionless units indicated in the labels. The solid line is the result from MD simulations, while the dot-dashed (blue) line and the (red) dashed line are the theoretical predictions from the stochastic and collisional models, respectively. The used values of $\xi_{0}$ and $\Delta$ are from Figs. 1 and 2.

density of the granular gas, the former leads to an expression proportional to $n^{-2 / 3}$.

\section{Time evolution of the temperature}

Once the values of the parameters $\xi_{0}$ and $\Delta$ defining each of the two models have been determined by fitting the steady temperature measured in the MD simulations of the confined granular gas, those values can be used to test the accuracy of the predictions for the time evolution of the temperature towards its steady value. In Figs. 3 and 4 the results obtained for two different values of the vibration velocity $v_{b}=10^{-3}(T(0) / m)^{1 / 2}$ and $v_{b}=10^{-2}(T(0) / m)^{1 / 2}$, respectively, are shown. The density,

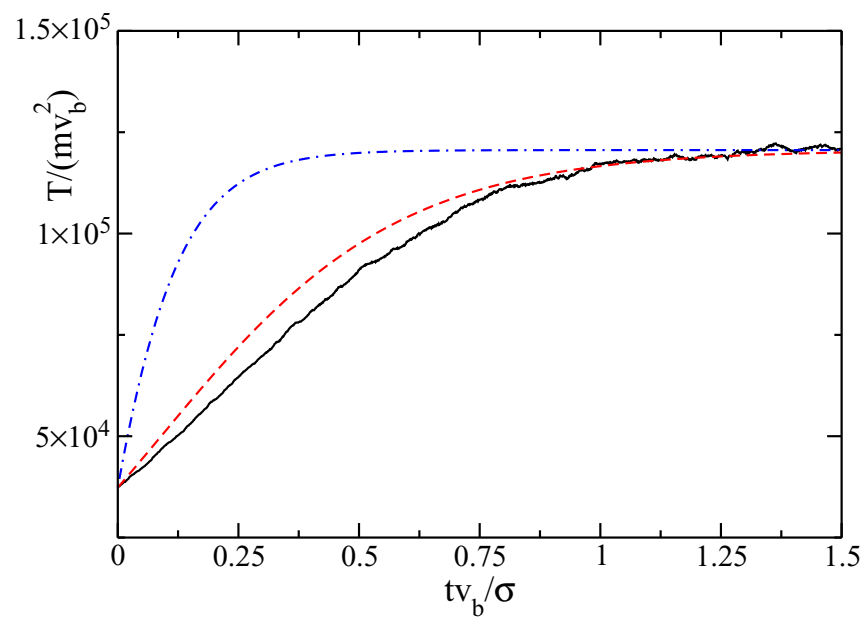

FIG. 4. (Color online) The same as described in the legend of Fig. 3 but now for a system with a vibrating wall with a velocity $v_{b}=10^{-2}(T(0) / m)^{1 / 2}$. the height $h$, and the coefficient of normal restitution $\alpha$ are the same as in Figs. 1 and 2. In the first case, the system monotonically cools towards the steady state, while in the latter the system heats while approaching stationarity. The solid lines are simulation data, the dot-dashed lines are the theoretical predictions obtained with the stochastic model, and the dashed lines are the predictions of the collisional model. It is seen that the prediction of the collisional model is much better than that of the stochastic model, in which the initial rate of variation of the temperature is much larger than in the MD simulations. This is not surprising since, as already indicated, the collisional model was specifically designed trying to mimic the horizontal dynamics of a quasi-two-dimensional system, while the stochastic model was formulated without addressing any particular system or state. In any case, it is worth mentioning that the stochastic model leads to predictions that in some cases are in good agreement with the results obtained for a vibrated gas of inelastic hard spheres or disks [40,41]. Similar results have been obtained for other values of the vibration velocity $v_{b}$, although the accuracy of the theoretical predictions gets worse as the velocity increases.

\section{THE STEADY VELOCITY DISTRIBUTION}

The steady one-particle velocity distribution in the horizontal plane, $f_{\mathrm{st}}(\boldsymbol{v})$, has also been measured in the MD simulations of the granular gas confined between two parallel plates. Taking into account the isotropy of the system in the horizontal plane, results will be presented in the following for the marginal velocity distribution $\varphi_{x}\left(c_{x}\right)$ defined by

$$
\varphi_{x}\left(c_{x}\right) \equiv \int_{-\infty}^{+\infty} d c_{y} \varphi(c)
$$

where $\varphi(c)$ is the scaled velocity distribution of the dimensionless velocity $\boldsymbol{c} \equiv \boldsymbol{v} / v_{0, \mathrm{st}}$, with $v_{0, \mathrm{st}} \equiv\left(2 T_{\mathrm{st}} / m\right)^{1 / 2}$,

$$
f_{\mathrm{st}}(\boldsymbol{v}) \equiv n v_{0, \mathrm{st}}^{-2} \varphi(c) \text {. }
$$

The simulation results indicate that the shape of the velocity distribution is very similar to a Gaussian. This leads to try to describe it by means of a truncated Sonine expansion. To lowest order, it has the form [15]

$$
\varphi_{x}\left(c_{x}\right) \approx \pi^{-1 / 2} e^{-c_{x}^{2}}\left[1+\frac{a_{2, \mathrm{st}}}{2}\left(\frac{3}{4}-3 c_{x}^{2}+c_{x}^{4}\right)\right] .
$$

The Sonine coefficient $a_{2, \mathrm{st}}$ is related with the fourth moment of the velocity distribution by

$$
a_{2, \mathrm{st}} \equiv \frac{\left\langle c^{4}\right\rangle}{2}-1
$$

with

$$
\left\langle c^{4}\right\rangle \equiv \int d c c^{4} \varphi(c) .
$$

In the simulation results being reported, $a_{2, \text { st }}$ has been measured using Eq. (21). In Fig. 5, the Sonine coefficient is plotted as a function of the coefficient of normal restitution for a system of 500 inelastic hard spheres with $v_{b}=5 \times$ $10^{-3}(T(0) / m)^{1 / 2}, n=0.02 \sigma^{-3}$, and $h=1.5 \sigma$. Two features of the simulation data must be emphasized. First, $a_{2, \text { st }}$ is positive in all the range of $\alpha$ investigated. Second, it is very 


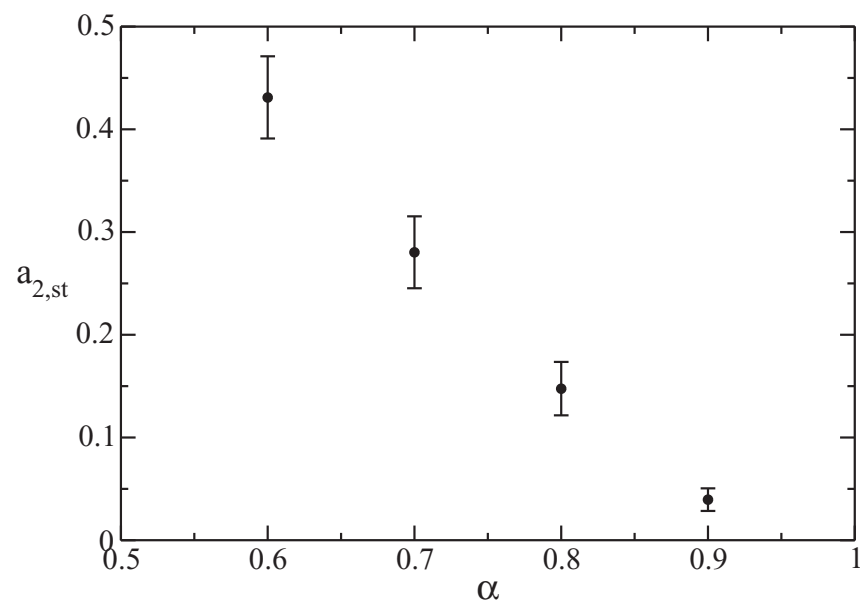

FIG. 5. MD simulation results for the Sonine coefficient $a_{2, \text { st }}$ of the steady velocity distribution function in the horizontal plane of the quasi-two-dimensional granular gas as a function of the coefficient or normal restitution $\alpha$. The velocity of the vibrating wall is $v_{b}=$ $5 \times 10^{-3}(T(0) / m)^{1 / 2}$, the density of the three-dimensional gas is $n=$ $0.02 \sigma^{-3}$, and the distance between the two confining walls is $h=$ $1.5 \sigma$.

small for moderate and weak inelasticity, say, $\alpha \gtrsim 0.8$, while it grows rather fast for stronger inelasticities. It is interesting to compare this behavior with the results obtained from the two models discussed in the previous section. The collisional model predicts [13] that the coefficient is negative and very small ( $\gtrsim-0.12)$ for all $\alpha$. On the other hand, the stochastic thermostat model leads to a coefficient that is negative and small for weak inelasticity, becoming positive for $\alpha \lesssim 0.7$ and increasing very slowly afterwards [4]. The fair conclusion is that none of the models is able to reproduce the qualitative behavior of the Sonine coefficient, but both accurately predict that the coefficient is very small in the quasielastic regime. To put this conclusion into proper context, it is important to stress that the theoretical predictions for $a_{2, \text { st }}$ have been obtained by using a linear approximation in which only terms to first order in $a_{2}$ are kept in the calculations $[4,13]$.

The dependence of $a_{2, \text { st }}$ on $v_{b}$ is analyzed in Fig. 6 for a system with $\alpha=0.8$ and the same values of the other parameters as in Fig. 5. No dependence on $v_{b}$ is observed. The same happens for other values of the restitution coefficient. This is consistent with the theoretical predictions following from both the stochastic model [2] and the collisional one [13], in which the steady Sonine coefficient is independent from $\xi_{0}$ and $\Delta$, respectively.

The fact that the Sonine coefficient $a_{2}$ is small does not guarantee by itself that the one-particle distribution function can be accurately approximated by a Gaussian. It could happen that contributions of higher-order cumulants be relevant and even that the Sonine expansion be divergent [42]. To see whether this is the case, in Fig. 7 the marginal one-particle distribution function $\varphi_{x}\left(c_{x}\right)$ measured by MD simulations is shown, again for $\alpha=0.8, n=0.02 \sigma^{-3}$, and $h=1.5 \sigma$. Also plotted are the Gaussian and the first Sonine approximation, Eq. (20), using the value of $a_{2, \text { st }}$ measured in the MD simulations. The agreement between the Sonine approximation and the actual velocity distribution is very good. Also notice that the deviation

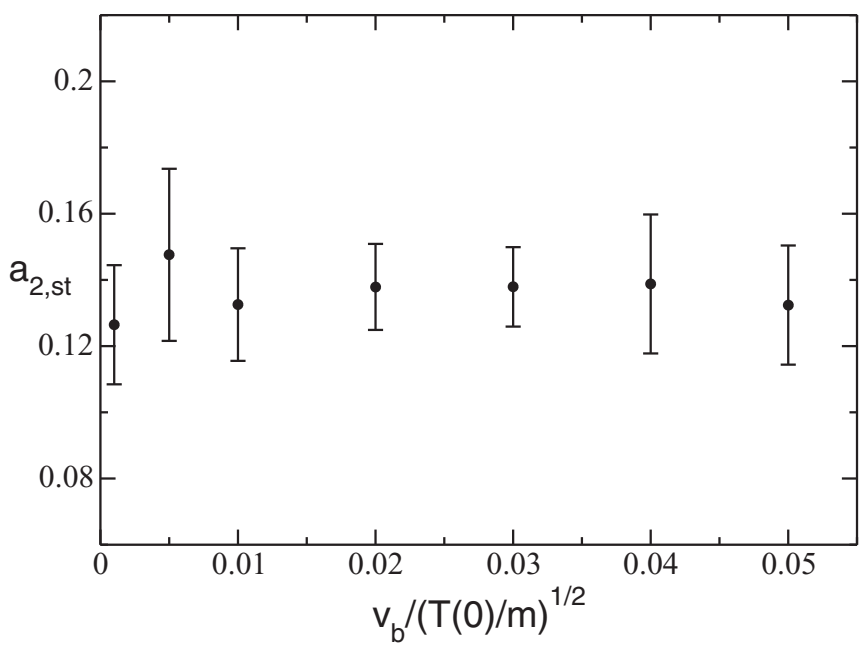

FIG. 6. MD simulation results for the Sonine coefficient $a_{2, \text { st }}$ of the steady velocity distribution function of the quasi-two-dimensional granular gas as a function of the dimensionless velocity of the vibrating wall. The coefficient of normal restitution is $\alpha=0.8$, the density of the three-dimensional gas is $n=0.02 \sigma^{-3}$, and the distance between the two confining walls is $h=1.5 \sigma$.

of the distribution from the Maxwell-Boltzmann one is clearly observed. In summary, the velocity distribution describing the horizontal motion of the particles is very well represented by Eq. (20), at least in thermal region, i.e., for values of $c$ of the order of unity. This is similar to what has been found for the thermostated model [43] and also for the collisional one [13].

\section{THE KOVACS-LIKE EFFECT IN THE COLLISIONAL MODEL}

In this section, the behavior of the collisional model when submitted to a protocol of the type considered by Kovacs [17]

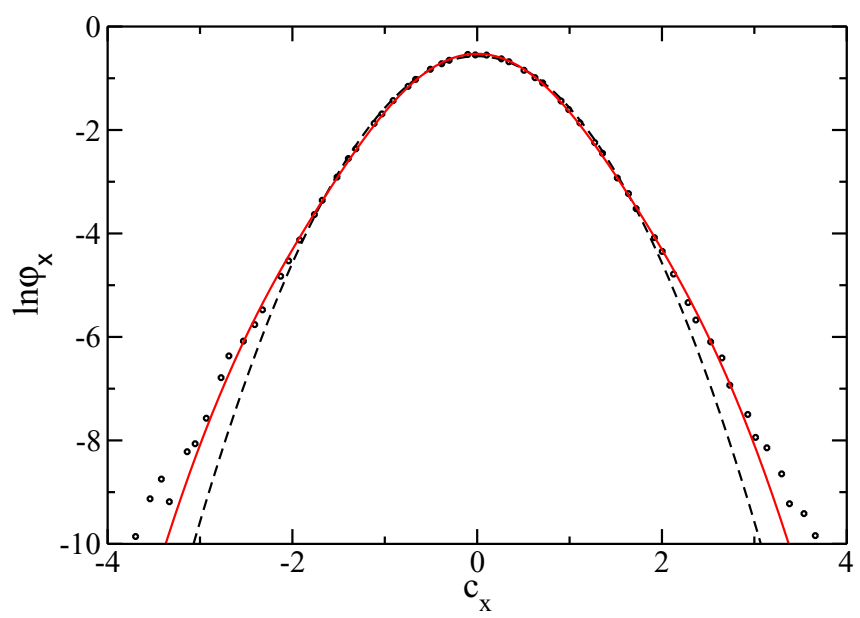

FIG. 7. (Color online) Logarithmic plot of the MD simulation values (black circles) of the marginal one-particle velocity distribution $\varphi_{x}\left(c_{x}\right)$ defined by Eq. (18). The (black) dashed line is the Gaussian and the (red) solid line is the Sonine approximation as given by Eq. (20), with the value of $a_{2 \text {,st }}$ measured also in the simulations. The parameters of the system are the same as described in the legend to Fig. 6 , and $v_{b}=10^{-3}(T(0) / m)^{1 / 2}$. 
will be investigated. A similar study for the stochastic thermostated model has been carried out recently [23]. Since the method of analysis used in that reference is not the same as the one employed here, in Appendix B the application of the present approximation to the stochastic model is briefly described. As expected, the conclusions reached are consistent with the results reported in Ref. [23].

In the context of kinetic theory, the existence of a macroscopic hydrodynamic regime, in which the time evolution of the temperature obeys a closed equation, requires that the one-particle distribution function of the system be normal, meaning that all its time dependence occurs through the temperature $[15,16]$. Then dimensional analysis leads to a scaling of the velocity distribution of the collisional model of the form

$$
f_{H}(\boldsymbol{v}, t)=n v_{0}(t)^{-2} \varphi\left(\boldsymbol{c}, \Delta^{*}\right),
$$

where $v_{0}(t)$ and $c$ are defined below Eq. (13) and in Eq. (15), respectively. The index $H$ indicates that we are restricting ourselves to homogenous hydrodynamic states. Consequently, if the first Sonine approximation (14) is employed, it follows that the dimensionless Sonine coefficient $a_{2}$ must also be normal, i.e.,

$$
a_{2}(t) \rightarrow \bar{a}_{2}(t)=\bar{a}_{2}\left(\frac{T(t)}{T_{\mathrm{st}}}\right) .
$$

Equivalently, the time-dependent temperature could have been scaled with $m \Delta^{2}$. The existence of a normal solution in which $a_{2}(t)$ is of the above form, and, therefore, of homogeneous hydrodynamics, has been established for both the stochastic model [44] and the collisional model [14].

Suppose a two-dimensional system of hard disks with the collision rule given by Eqs. (10) and (11) and with a characteristic speed $\Delta=\Delta_{1}$. The system is in the steady state, so its temperature, $T_{1, \mathrm{st}}$, is given by

$$
\frac{\Delta_{1}}{v_{01, \mathrm{st}}}=\Delta_{\mathrm{st}}^{*}
$$

where

$$
v_{01, \mathrm{st}} \equiv\left(\frac{2 T_{1, \mathrm{st}}}{m}\right)^{1 / 2}
$$

and $\Delta_{\text {st }}^{*}$ fulfils the equation [see Eq. (12)]

$$
\zeta^{*}\left(\alpha, \Delta_{\mathrm{st}}^{*}\right)=0
$$

Here, the dimensionless hydrodynamic rate of variation of the energy,

$$
\begin{aligned}
\zeta^{*}\left(\alpha, \Delta^{*}\right) \equiv & (2 \pi)^{1 / 2}\left[\frac{1-\alpha^{2}}{2}\left(1+\frac{3 \bar{a}_{2}\left(\Delta^{*}\right)}{16}\right)\right. \\
& \left.-\alpha\left(\frac{\pi}{2}\right)^{1 / 2} \Delta^{*}-\left(1-\frac{\bar{a}_{2}\left(\Delta^{*}\right)}{16}\right) \Delta^{* 2}\right]
\end{aligned}
$$

has been introduced. Then, at a given moment, the characteristic speed is changed to $\Delta_{2}<\Delta_{1}$. The steady temperature $T_{2 \text {,st }}$ corresponding to the new value of the characteristic speed will be given by

$$
\left(\frac{2 T_{2, \mathrm{st}}}{m}\right)^{1 / 2}=\frac{\Delta_{2}}{\Delta_{\mathrm{st}}^{*}}<\frac{\Delta_{1}}{\Delta_{\mathrm{st}}^{*}}=\left(\frac{2 T_{1, \mathrm{st}}}{m}\right)^{1 / 2} .
$$

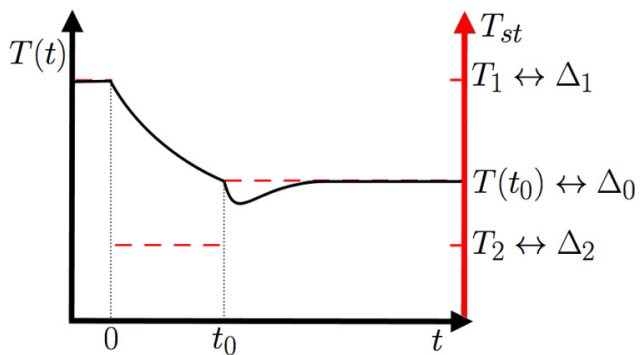

FIG. 8. (Color online) Sketch of the protocol followed in the Kovacs-like experiment described in the main text. Initially, the system is in the steady state corresponding to a value $\Delta_{1}$ of the characteristic speed parameter, with $T_{1}$ as its temperature. Then, at $t=0$, the speed parameter is suddently changed to $\Delta_{2}<\Delta_{1}$, and the system relaxes towards a new steady temperature $T_{2}<T_{1}$. At $t=t_{0}$, when the temperature of the system is $T\left(t_{0}\right)$, the characteristic speed is again suddently changed, now to a value $\Delta_{0}$ such that $T\left(t_{0}\right)$ is the steady temperature for it. The solid line describes the time evolution of the temperature of the system, assuming that it exhibits a negative hump before tending again towards its steady value.

Consequently, the system will monotonically cool towards $T_{2 \text {,st }}$, at least after some nonhydrodynamic initial transient. Let us denote the time-dependent temperature once in the hydrodynamic regime by $T(t)$. The one-particle distribution function of the system describing its time evolution in the first Sonine approximation is given by Eq. (14) with

$$
\begin{aligned}
a_{2}(t) & =\bar{a}_{2}\left(\alpha, \Delta_{2}^{*}(t)\right), \\
\Delta_{2}^{*}(t) & \equiv \frac{\Delta_{2}}{v_{0}(t)} .
\end{aligned}
$$

Consider next a later time $t_{0}$ where $T_{0}=T\left(t_{0}\right)$ is the temperature of the system at that time. The cooling rate at the same moment is

$$
\zeta\left(\alpha, \Delta_{2}^{*}\left(t_{0}\right)\right)=n \sigma v_{0}\left(t_{0}\right) \zeta^{*}\left(\alpha, \Delta_{2}^{*}\left(t_{0}\right)\right) .
$$

As indicated above, the system is cooling and therefore it is

$$
\zeta^{*}\left(\alpha, \Delta_{2}^{*}\left(t_{0}\right)\right)>0 .
$$

There is a value $\Delta_{0}$ of the characteristic speed for which

$$
\frac{\Delta_{0}}{v_{0}\left(t_{0}\right)}=\Delta_{\mathrm{st}}^{*}
$$

so $T_{0}$ is precisely the steady temperature for $\Delta=\Delta_{0}$. In the experiment being described, the value of the characteristic speed is instantaneously modified at $t=t_{0}$ to the value $\Delta_{0}$, defined above. The protocol applied to the system is sketched in Fig. 8. Therefore, at $t=t_{0}$, the cooling rate of the system changes to

$$
\zeta_{+}\left(\alpha, t_{0}\right)=n \sigma v_{0}\left(t_{0}\right) \zeta_{+}^{*}\left(\alpha, t_{0}\right)
$$

with

$$
\begin{aligned}
\zeta_{+}^{*}\left(\alpha, t_{0}\right)= & (2 \pi)^{1 / 2}\left\{\frac{1-\alpha^{2}}{2}\left[1+\frac{3 \bar{a}_{2}\left(\Delta_{2}^{*}\left(t_{0}\right)\right)}{16}\right]\right. \\
& \left.-\alpha\left(\frac{\pi}{2}\right)^{1 / 2} \Delta_{\mathrm{st}}^{*}-\left[1-\frac{\bar{a}_{2}\left(\Delta_{2}^{*}\left(t_{0}\right)\right)}{16}\right] \Delta_{\mathrm{st}}^{* 2}\right\} .
\end{aligned}
$$




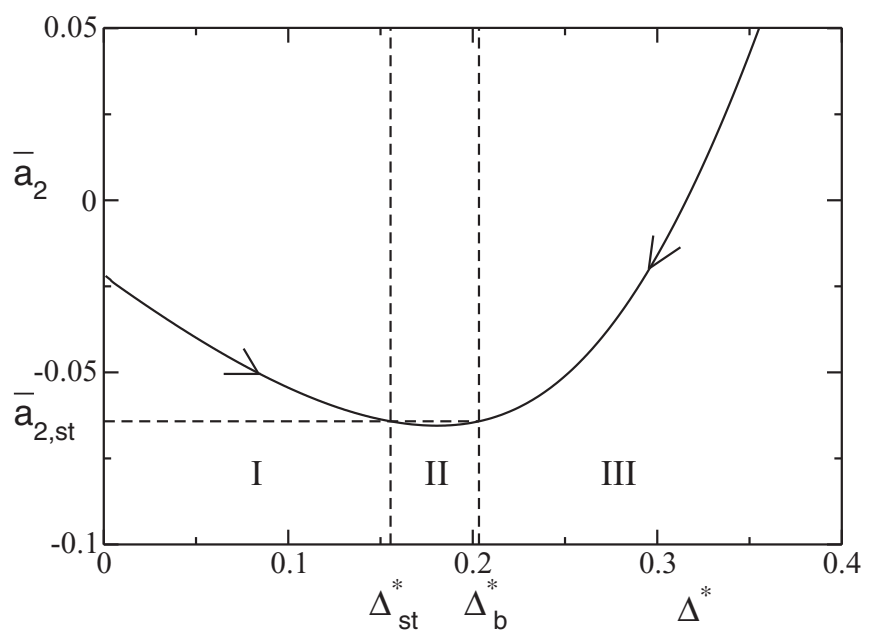

FIG. 9. Hydrodynamic Sonine coefficient $\bar{a}_{2}$, as a function of the dimensionless characteristic speed $\Delta^{*}$ for a system with a coefficient of normal restitution $\alpha=0.8$. Shown in the figure are the steady values of $a_{2}$ and $\Delta^{*}, a_{2, \mathrm{st}}$ and $\Delta_{\mathrm{st}}^{*}$, respectively. Also plotted is the value $\Delta_{b}^{*}$, defined by Eq. (38). The arrows on the curve indicate the way in which it is described as the system approaches its steady state. For constant $\Delta$, interval I corresponds to cooling and intervals II and III to heating. If the value of $a_{2}$ at the moment $t=t_{0}$ in which $\Delta$ is modified so $\Delta^{*}\left(t_{0}\right)=\Delta_{\mathrm{st}}^{*}$, lies in regions I or II, the system keeps on cooling and heating, respectively. On the other hand, if it lies in region III the system instantaneously changes from heating to cooling (rebound effect).

Here it has been taken into account that $\Delta_{0}$ has been chosen such that $\Delta^{*}=\Delta_{\mathrm{st}}^{*}$ at $t=t_{0}$ and this shows up in the dynamics of the system, but the initial form of the distribution function for the subsequent evolution of the system is of course the one corresponding to $\bar{a}_{2}\left(\Delta_{2}^{*}\left(t_{0}\right)\right)$. Using Eq. (27), this expression can be rewritten as

$$
\begin{aligned}
\zeta_{+}^{*}\left(\alpha, t_{0}\right)= & \frac{(2 \pi)^{1 / 2}}{16}\left[\frac{3\left(1-\alpha^{2}\right)}{2}+\Delta_{\mathrm{st}}^{* 2}\right] \\
& \times\left[\bar{a}_{2}\left(\Delta_{2}^{*}\left(t_{0}\right)\right)-\bar{a}_{2}\left(\Delta_{\mathrm{st}}^{*}\right)\right] .
\end{aligned}
$$

It follows that the cooling rate $\zeta_{+}\left(\alpha, t_{0}\right)$ is positive if $\bar{a}_{2}\left(\Delta_{2}^{*}\left(t_{0}\right)\right)>\bar{a}_{2}\left(\Delta_{\text {st }}^{*}\right)$ and negative if $\bar{a}_{2}\left(\Delta_{2}^{*}\left(t_{0}\right)\right)<\bar{a}_{2}\left(\Delta_{\text {st }}^{*}\right)$. It only vanishes if $\bar{a}_{2}\left(\Delta_{2}^{*}\left(t_{0}\right)\right)=\bar{a}_{2}\left(\Delta_{\text {st }}^{*}\right)$, i.e., the system was at $t=t_{0}$ in the steady state. In Fig. 9, the hydrodynamic Sonine coefficient $\bar{a}_{2}$ is plotted as a function of $\Delta^{*}$ for a system with $\alpha=0.8$ [14]. The steady value $\Delta_{\text {st }}^{*}$ is indicated. In a cooling experiment, as the one occurring when $\Delta$ is changed to $\Delta_{2}$, the value of $\Delta^{*}$ is increasing towards its steady value and $\bar{a}_{2}$ is decreasing towards $\bar{a}_{2, \mathrm{st}}$. It follows that it is always $\bar{a}_{2}\left(\Delta_{2}^{*}\left(t_{0}\right)\right)>\bar{a}_{2}\left(\Delta_{\text {st }}^{*}\right)$. This conclusion holds as long as the dependence of $\bar{a}_{2}$ on $\Delta^{*}$ is similar to the one illustrated in Fig. 9. Actually, this happens in the collisional model for all the physical values of the restitution coefficient, $0<\alpha \leqslant 1$.

Consider next the same experiment, but this time with $\Delta_{1}<$ $\Delta_{2}$, i.e., the system is increasing its temperature monotonically when $\Delta$ is instantaneously changed from $\Delta_{2}$ to $\Delta_{0}$ at $t=t_{0}$. In other words, its cooling rate is negative just before the change. What happens just after the change? If $\bar{a}_{2}\left(\Delta^{*}\left(t_{0}\right)\right)>\bar{a}_{2}\left(\Delta_{\mathrm{st}}^{*}\right)$, the cooling rate is positive after the change and the system

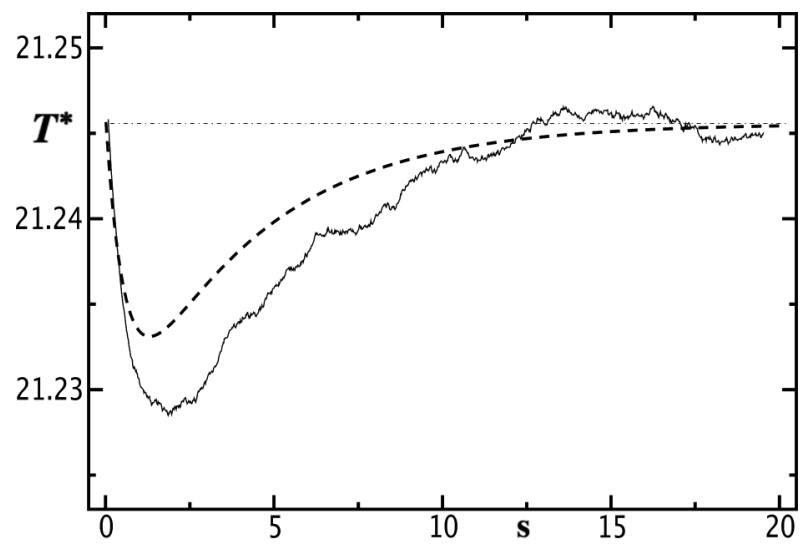

FIG. 10. The Kovacs effect in a system under cooling. Temperature $T^{*}$ and time $s$ are measured in the dimensionless units indicated in the main text. The characteristic speeds verify $\Delta_{2}=0.5 \Delta_{0}$, where $\Delta_{2}$ is the speed in the former (no plotted) evolution, and $\Delta_{0}$ is the speed in the final relaxation starting at the steady temperature. The dashed line is the theoretical prediction given by the solution of Eqs. (A1) and (A2), while the solid line has been obtained by the DSMC method.

is cooling; its temperature decreases in time. On the other hand, if $\bar{a}_{2}\left(\Delta^{*}\left(t_{0}\right)\right)<\bar{a}_{2}\left(\Delta_{\text {st }}^{*}\right)$, the system will keep on heating. The former case can be understood as a rebound effect in the evolution of the temperature. The above discussion can expressed alternatively as follows. Define $\Delta_{b}^{*}$ as the value $\Delta^{*}>\Delta_{\text {st }}^{*}$ verifying

$$
\bar{a}_{2}\left(\Delta_{b}^{*}\right)=\bar{a}_{2, \mathrm{st}}
$$

If $\Delta_{2}^{*}\left(t_{0}\right)>\Delta_{b}^{*}$, then there is rebound effect of the temperature, while if $\Delta_{\mathrm{st}}^{*}<\Delta_{2}^{*}\left(t_{0}\right)<\Delta_{b}^{*}$, then there is no such effect, and the temperature continues increasing after the modification of $\Delta$. In Fig. 9, the several intervals for $a_{2}\left(\Delta_{2}^{*}\left(t_{0}\right)\right)$, defining the qualitative behavior of the system after modification of the characteristic speed $\Delta$ are indicated.

The fact that the temperature of the system after the change of the characteristic speed does not remain in its steady value but deviates initially from it to decay afterwards can be considered as reminiscent of the Kovacs effect exhibited by molecular system in their equilibrium relaxation [17]. Evidently, this happens because the system is not in the steady state, in spite of having the steady temperature. In the context of kinetic theory, this implies that its velocity distribution function is not fully determined by the instantaneous temperature, i.e., it is not normal.

In the Sonine approximation for the one-particle velocity distribution given by Eq. (14), the time evolution of the temperature in an homogeneous system can be described by means of a pair of coupled differential equations. Assuming that the Sonine coefficient $a_{2}$ and its time derivative are small, the equations can be written in the form given in Appendix A, Eqs. (A1) and (A2). In the following, numerical solutions of those equations will be reported in order to verify and also quantify the conclusions reached in the discussion carried out above. In Fig. 10 the dimensionless temperature $T^{*}(t) \equiv$ $T(t) / m \Delta^{2}$ is plotted as a function of the dimensionless time $s$ given by $d s \equiv v_{0}(t) n \sigma d t$, where $v_{0}$ is the thermal velocity 


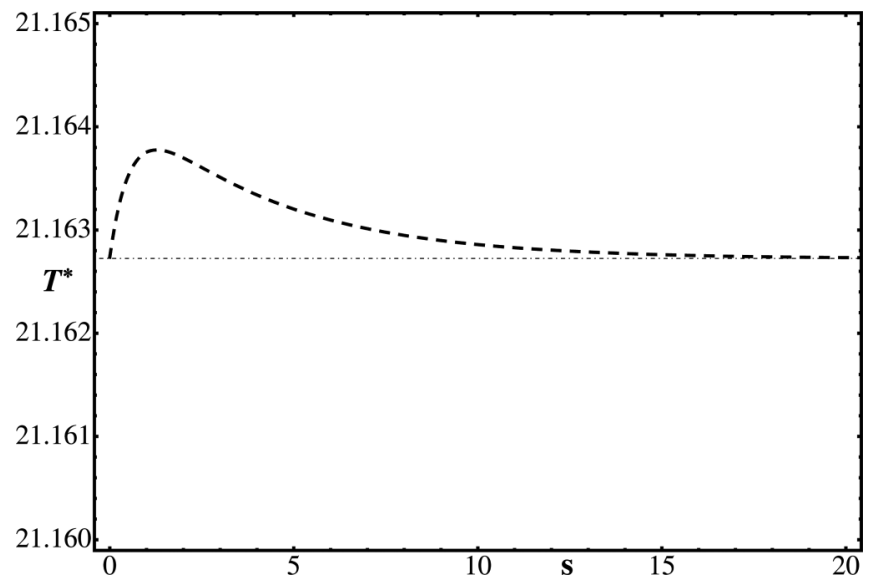

FIG. 11. The Kovacs effect without rebound in a system under heating. Temperature $T^{*}$ and time $s$ are measured in the dimensionless units indicated in the main text. The characteristic speeds verify $\Delta_{2}=$ $1.2 \Delta_{0}$, where $\Delta_{2}$ is the speed in the former evolution, and $\Delta_{0}$ is the speed in the final relaxation starting at its steady temperature. The dashed curve is the solution of the evolution equations given in the Appendix.

defined above Eq. (14). The time scale $s$ is proportional to the accumulated number of collisions per particle in the system, and the origin $s=0$ has been chosen for $t=t_{0}$, so only the evolution after putting $\Delta=\Delta_{0}$ is plotted. The values of the characteristic speeds of the process are related by $\Delta_{0}=2 \Delta_{2}$. This relation fully specify the solution for $t>t_{0}$, as discussed in Appendix A. Since $\Delta_{0}>\Delta_{2}$, the system was cooling before the change of the characteristic speed to $\Delta_{0}$. As predicted by the above discussion, the system keeps cooling after the change, later approaching its steady value from below.

Since the model predictions for the Sonine coefficient $a_{2}$ qualitatively disagree with the MD results, it is clear that the effect being analyzed now must differ in the model and in the original quasi-two-dimensional confined gas. For this reason, it seems more illuminating to consider the numerical results obtained by the direct simulation Monte Carlo method (DSMC) applied to the collisional model than MD results. The DSMC method is a particle simulation algorithm designed to mimic the dynamics of a low-density gas [45,46], i.e., a gas obeying the Boltzmann equation. This simulation method has been extensively applied to granular gases, and it will not be described here. Let us only mention that the homogeneity of the system being simulated can be exploited to improve the accuracy of the results [47]. The number of particles employed in the simulations is $N=1000$ and the reported results have been averaged over $5 \times 10^{4}$ trajectories. Included in Fig. 10 are the DSMC results for a system with $\Delta_{2}=0.5(T(0) / m)^{1 / 2}$ and $\Delta_{0}=(T(0) / m)^{1 / 2}$, where $T(0)$ is a reference initial temperature. The agreement between the simulation results and the theoretical prediction can be considered as satisfactory, taking into account the approximations involved in the derivation of Eqs. (A1) and (A2). Note that the plotted curves start at $t=t_{0}$ $(s=0)$, at which the characteristic speed is changed from $\Delta_{2}$ to $\Delta_{0}$.

The behavior of the system in heating experiments is illustrated in Figs. 11 and 12. The former is for $\Delta_{2}=1.2 \Delta_{0}$,

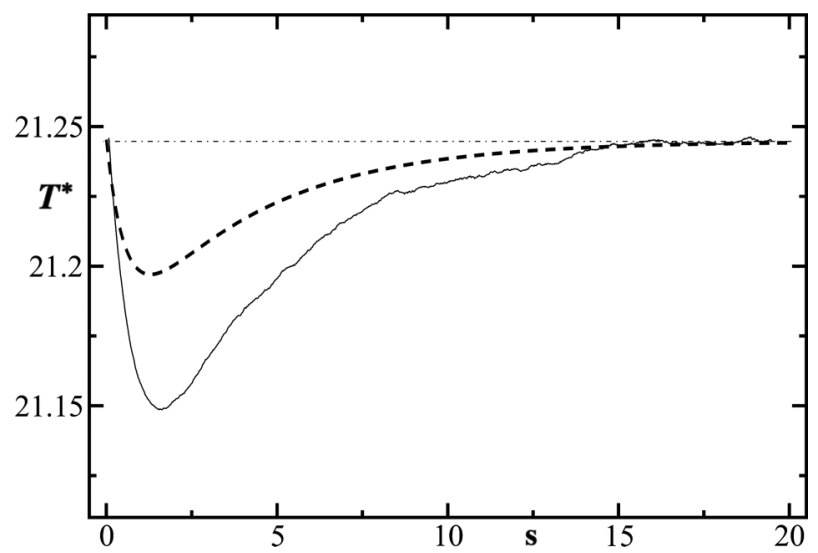

FIG. 12. The Kovacs effect with rebound in a system under heating. Temperature $T^{*}$ and time $s$ are measured in the dimensionless units indicated in the main text. The characteristic speeds verify $\Delta_{2}=2 \Delta_{0}$, where $\Delta_{2}$ is the speed in the former evolution, and $\Delta_{0}$ is the speed in the final relaxation starting at its steady temperature. The dashed line is the theoretical prediction given by the solution of Eqs. (A1) and (A2), while the solid line has been obtained by the DSMC method. Note that the temperature of the system was increasing for $s<0$.

while the latter is for $\Delta_{2}=2 \Delta_{0}$. In both cases the system is heating just before $\Delta$ is modified from $\Delta_{2}$ to $\Delta_{0}$. The difference between them is that the value of $a_{2}\left(t_{0}\right)=\bar{a}_{2}\left(\Delta_{2} \Delta_{\text {st }}^{*} / \Delta_{0}\right)$ at that time is in the first case smaller than $\bar{a}_{2 \text {,st }}$, while it is larger in the second one. In agreement with the discussion carried out above, the temperature keeps on increasing just after the change of the peculiar velocity to $\Delta_{0}$ in the first experiment, while it decreases (rebound effect) in the second one. Note that only the evolution for $t>t_{0}$ is plotted. DSMC simulation results are shown only for one of the cases, namely for $\Delta_{2}=2(T(0) / m)^{1 / 2}$ and $\Delta_{0}=(T(0) / m)^{1 / 2}$. This is because the values of $\bar{a}_{2}\left(\Delta^{*}\right)$ for $\Delta_{s t}^{*}<\Delta^{*}<\Delta_{b}^{*}$ are very close to $\bar{a}_{2, \mathrm{st}}$, as it is seen in Fig. 9. As a consequence, when starting with a value of $a_{2}$ within the interval II in Fig. 9, the effect is very weak, and hard to measure in the DSMC simulations because of the statistical uncertainties. The different scales used on the vertical axis of Figs. 11 and 12 must be stressed.

Similar results have been obtained for other relations of the charateristic speeds and also for $\alpha=0.9$, then expecting the same degree of agreement between theory and DSMC simulations over a wide range of parameters.

\section{CONCLUDING REMARKS}

In the first part of this paper, the accuracy of the collisional model introduced by Brito et al. [10] to describe the homogeneous two-dimensional dynamics of a confined gas of inelastic hard spheres has been investigated. The phenomenological speed parameter involved in the formulation of the model has been determined by fitting the MD simulation values of the steady temperature. Next, the time evolution of the temperature when relaxing towards the steady state predicted by the model using that value has been compared with the simulation results, and an acceptable good agreement has been found. Moreover, the fitted characteristic speed of the model is proportional 
to the vibration velocity of the walls in the original system, what is consistent with its physical interpretation. A similar program has been carried out with a thermostated random model, often used in the literature to generate a steady state of granular system [2,4,36-39], getting a worse agreement with the simulation data. Also the one-particle velocity distribution function of the system in the steady state has been studied. In the simulations, as well as in the two models considered, the steady distribution turns out to be quite accurately well described by a Gaussian plus a small correction given by the first Sonine approximation. The coefficient measuring the magnitude of the correction term only depends on the value of the coefficient of normal restitution, but not of the parameter measuring the rate of injection of energy in the system.

The first Sonine approximation also describes accurately the homogeneous hydrodynamics of the collisional model [14]. In this context, it must be emphasized that, although the Sonine coefficient be quantitatively small, its existence and dependence on time through the temperature is essential for the possibility of a hydrodynamic description. The second part of the paper deals with a memory effect in the time evolution of the temperature in the collisional model that is also well captured by the first Sonine approximation. The characteristic speed parameter is suddenly changed while the system is relaxing towards its steady state, so that the latter is modified. This is done in such a way that the system is instantaneously at the steady temperature corresponding to the new speed. Then, the posterior evolution of the temperature exhibits a highly nonmonotonic behavior in which the temperature of the system initially deviates from its long time steady value. This behavior somehow resembles the Kovacs effect occurring in the relaxation to equilibrium of molecular systems [17]. The analysis presented here largely benefits from considering that, when the characteristic speed is modified, the system was evolving accordingly with the hydrodynamic law. This allows to determine its distribution function at that moment just from the knowledge of the temperature. Of course, afterwards this property is lost, the distribution function is not determined by the temperature (i.e., it is not normal) and the system is not in the hydrodynamic regime.

Under the above hydrodynamic initial condition, it is easy to explain the existence of memory effects in the collisional model and to predict the qualitative behavior of the temperature of the system after the jump of the characteristic speed, i.e., whether the temperature keeps on varying monotonically or there is some kind of rebound effect, with the temperature rate changing sign at the moment of the jump. Moreover, quantitative predictions are given by the (numerical) solutions of two coupled first order differential equations. A good agreement has been found between the theoretical predictions and simulation values obtained by the direct simulation Monte Carlo method. As already mentioned, a similar Kovacs-like effect has been studied in the stochastic thermostat model described in Sec. II [23]. For completeness, a discussion of the effect in the same model but using the approach developed in the previous section is briefly presented in Appendix B. The main difference between the behaviors of the collisional model and the stochastic one, is that the latter shows rebound in the temperature for weak dissipation (small inelasticity) and no rebound for strong dissipation (large inelasticity), independently of whether the system was cooling of heating at the time of the final jump of the parameter controlling the steady temperature of the granular system.

The reported results show that highly nonlinear and nonhydrodynamic evolution of a system can be quite easily understood by means of simple kinetic theory arguments. Quite peculiarly, here this has been done by taking advantage of choosing the initial evolution state as being described by hydrodynamics. Moreover, the analysis suggests the possibility of experimentally observing the nonlinear relaxation of the Kovacs-like effect in a purely mechanical system, namely the confined quasi-two-dimensional granular gas submitted to vertical vibration. The restriction to initial hydrodynamic states seems something easily implementable. Nevertheless, some caution is needed in extrapolating the results for the models to a real system, and even to the confined quasitwo-dimensional system of ideal inelastic hard spheres. It might happen that the detailed mechanism responsible for the presence of memory effects in a confined system differs from those analyzed in this paper for the two models studied.

\section{ACKNOWLEDGMENTS}

This research was supported by the Ministerio de Economía y Competitividad (Spain) through Grant No. FIS2011-24460 (partially financed by FEDER funds).

\section{APPENDIX A: TIME EVOLUTION OF THE TEMPERATURE IN THE SONINE APPROXIMATION}

By taking moments in the Boltzmann equation and considering the Sonine approximation given by Eq. (14), the following pair of coupled first order differential equations are obtained:

$$
\begin{aligned}
\frac{\partial \Delta^{*}}{\partial \tau} & =\frac{(2 \pi)^{1 / 2}}{8}\left[A_{0}+A_{1} a_{2}(t)\right], \\
\frac{\partial a_{2}}{\partial \tau} & =\frac{(2 \pi)^{1 / 2}}{8 \Delta^{*}(t)}\left\{\left[4 A_{0}+B_{0}\right]+\left[4\left(A_{0}+A_{1}\right)+B_{1}\right] a_{2}(t)\right\},
\end{aligned}
$$

$$
\begin{aligned}
A_{0}\left(\alpha, \Delta^{*}\right)= & 4\left[\frac{1-\alpha^{2}}{2}-\left(\frac{\pi}{2}\right)^{1 / 2} \alpha \Delta^{*}-\Delta^{* 2}\right], \\
A_{1}\left(\alpha, \Delta^{*}\right)= & \frac{1}{4}\left[\frac{3\left(1-\alpha^{2}\right)}{2}+\Delta^{* 2}\right], \\
B_{0}\left(\alpha, \Delta^{*}\right)= & (2 \pi)^{1 / 2}\left(5+3 \alpha^{2}+4 \Delta^{* 2}\right) \alpha \Delta^{*}-3+4 \Delta^{* 4} \\
& +\alpha^{2}+2 \alpha^{4}-4\left(1-\alpha^{2}-2 \Delta^{* 2}\right) \\
& +2 \Delta^{* 2}\left(1+6 \alpha^{2}\right), \\
B_{1}\left(\alpha, \Delta^{*}\right)= & \left(\frac{\pi}{2}\right)^{1 / 2}\left[2-4(1-\alpha)+7 \alpha+3 \alpha^{3}\right] \Delta^{*} \\
& -\frac{1}{16}\left\{85+4 \Delta^{* 4}-18\left(3+2 \alpha^{2}\right) \Delta^{* 2}\right. \\
& -\left(32+87 \alpha+30 \alpha^{3}\right) \alpha \\
& \left.-4\left[6 \Delta^{* 2}-(1+\alpha)(31-15 \alpha)\right]\right\} .
\end{aligned}
$$



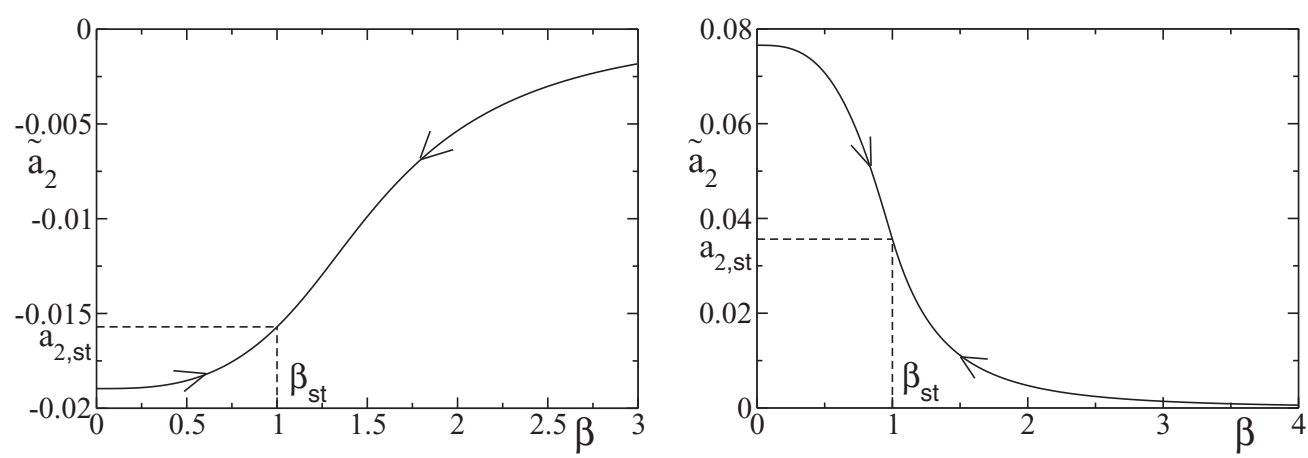

FIG. 13. Kurtosis in the hydrodynamic regime, $\tilde{a}_{2}$, as a function of the dimensionless parameter, $\beta$. The left figure is for $\alpha=0.95$ and the right one for $\alpha=0.5$. The stationary value of the kurtosis, $a_{2, s t}$, and of the dimensionless parameter, $\beta_{s}$, is also shown.

In the above expressions, a dimensionless time scale,

$$
\tau \equiv \Delta n \sigma t
$$

has been introduced. The general method leading to Eqs. (A1) and (A2) has been discussed in Ref. [14] and it will be not repeated in detail here. Let us only indicate that the former equation is obtained by multiplying the Boltzmann equation by the square of the velocity of the particles, integrating afterwards over it, while for the latter equation, one has to multiply the Boltzmann equation by the fourth power of the velocity before integrating. Moreover, upon deriving Eq. (A2) terms quadratic in $a_{2}$ have been neglected.

All the possible dependence of the solutions of Eqs. (A1) and (A2) on the characteristic speeds $\Delta_{2}$ and $\Delta_{0}$ occurs through the initial conditions. Let us focus on the evolution of the system after $\Delta$ is instantaneously changed from $\Delta_{2}$ to $\Delta_{0}$ at $t=t_{0}$. The fact that the system is in the hydrodynamic regime just before the change, and the way in which $\Delta_{0}$ is chosen have a relevant consequence. The initial conditions to be considered are given by

$$
\begin{aligned}
& \Delta^{*}\left(t=t_{0}\right)=\Delta_{\mathrm{st}}^{*}, \\
& a_{2}\left(t=t_{0}\right)=\bar{a}_{2}\left[\Delta_{2}^{*}\left(t_{0}\right)\right]=\bar{a}_{2}\left(\frac{\Delta_{2}}{\Delta_{0}} \Delta_{\mathrm{st}}^{*}\right) .
\end{aligned}
$$

It follows that the solution, and therefore the evolution of the temperature, is fully specified by the ratio of the characteristic speeds $\Delta_{0} / \Delta_{2}$, being independent of each of them separately. A similar argument can be applied to the previous evolution of $\Delta^{*}$ with $\Delta=\Delta_{2}$.

\section{APPENDIX B: THE KOVACS-LIKE EFFECT IN THE STOCHASTIC THERMOSTAT MODEL}

A similar analysis to the one made in Sec. IV for the collisional model, will be carried out in this Appendix for the stochastic thermostat case. The same notation as in the main text is used.

In the stochastic model, the evolution equation for the temperature in the hydrodynamic regime and for $d=2$ is [48]

$$
\frac{d T(t)}{d t}=-n \sigma v_{0}(t) \tilde{\zeta}(t) T(t),
$$

where $v_{0}(t)$ was defined bellow Eq. (13) and $\tilde{\zeta}(t)$ is the dimensionless rate of change of the temperature, which in the first Sonine approximation is given by

$$
\tilde{\zeta}(t)=\left(\frac{\pi}{2}\right)^{1 / 2}\left(1-\alpha^{2}\right)\left\{1-\beta^{3}+\frac{3}{16}\left[\tilde{a}_{2}(\beta)-a_{2, \mathrm{st}} \beta^{3}\right]\right\} .
$$

It is worth to stress that, in order to discuss the Kovacs-like effect, it is not enough to consider the Gaussian approximation leading to Eq. (6), although the latter provides an accurate description for the time evolution of the temperature in the stochastic model. In Eq. (B2), the dimensionless parameter $\beta(t) \equiv v_{0, \text { st }}\left(\xi_{0}\right) / v_{0}(t)$, with $v_{0, \text { st }}\left(\xi_{0}\right)$ being the steady value of $v_{0}(t)$, has been introduced. This parameter plays in the stochastic model a similar role to $\Delta^{*}$ in the context of the collisional model. The quantities $\tilde{a}_{2}(\beta)$ and $a_{2, \mathrm{st}} \equiv \tilde{a}_{2}\left(\beta_{s, t}\right)$ are the kurtosis in the hydrodynamic and stationary regimes, respectively [44].

Performing exactly the same analysis as in Sec. IV, but replacing the jumps in $\Delta$ by jumps in $\xi_{0}$, it is found that the rate of change of the temperature just after the last jump to $\xi_{0}^{0}$ is

$$
\tilde{\zeta}_{+}\left(t_{0}\right)=\left(\frac{\pi}{2}\right)^{1 / 2} \frac{3\left(1-\alpha^{2}\right)}{16}\left[\tilde{a}_{2}\left(\beta_{2}\left(t_{0}\right)\right)-a_{2, \mathrm{st}}\right],
$$

where $\beta_{2}\left(t_{0}\right)=v_{0, \mathrm{st}}\left(\xi_{0}^{0}\right) / v_{0}\left(t_{0}\right)$. The explicit expression for the function $\tilde{a}_{2}(\beta)$ was calculated in [44] and it will be not reproduced here. In Fig. 13, $\tilde{a}_{2}$ is plotted as a function of the parameter $\beta$ for $\alpha=0.95$ and $\alpha=0.5$, respectively. The qualitative behavior of $\tilde{a}_{2}$ for small inelasticities $(0.7 \lesssim \alpha<1)$ and strong inelasticities $(0<\alpha \lesssim 0.7)$ is rather different and it is well illustrated by the particular cases shown in Fig. 13).

Taking into account Eq. (B3) and the shape of the function $\tilde{a}_{2}(\beta)$, it is easily seen that, for small inelasticities (i.e., if $\left.a_{2, \mathrm{st}}<0\right)$ the Kovac's effect is always normal since $\tilde{a}_{2}(\beta)$ is a decreasing function, while the is anomalous for strong inelasticities (i.e., if $a_{2 \text { st }}>0$ ), because $\tilde{a}_{2}(\beta)$ now decreases. This behavior is consistent with the results reported in Ref. [23]. 
[1] I. Goldhirsch, Annu. Rev. Fluid Mech. 35, 267 (2003).

[2] T. P. C. van Noije and M. H. Ernst, Granular Matter 1, 57 (1998).

[3] A. Puglisi, V. Loreto, U. Marini Bettolo Marconi, and A. Vulpiani, Phys. Rev. E 59, 5582 (1999).

[4] I. Pagonabarraga, E. Trizac, T. P. C. van Noije, and M. H. Ernst, Phys. Rev. E 65, 011303 (2001).

[5] J. S. Olafsen and J. S. Urbach, Phys. Rev. Lett. 95, 098002 (2005).

[6] N. Rivas, S. Ponce, B. Gallet, D. Risso, R. Soto, P. Cordero, and N. Mújica, Phys. Rev. Lett. 106, 088001 (2011).

[7] F. Pacheco-Vázquez, G. A. Caballero-Robledo, and J. C. RuizSuárez, Phys. Rev. Lett. 102, 170601 (2009).

[8] G. Castillo, N. Mújica, and R. Soto, Phys. Rev. Lett. 109, 095701 (2012).

[9] N. V. Brilliantov and T. Pöschel, Kinetic Theory of Granular Gases (Oxford University Press, Oxford, 2004).

[10] R. Brito, D. Risso, and R. Soto, Phys. Rev. E 87, 022209 (2013).

[11] J. J. Brey, J. W. Dufty, and A. Santos, J. Stat. Phys. 87, 1051 (1997).

[12] T. P. C. van Noije, M. H. Ernst, and R. Brito, Physica A 251, 266 (1998).

[13] J. J. Brey, M. I. García de Soria, P. Maynar, and V. Buzón, Phys. Rev. E 88, 062205 (2013).

[14] J. J. Brey, P. Maynar, M. I. García de Soria, and V. Buzón, Phys. Rev. E 89, 052209 (2014).

[15] P. Résibois and M. de Leener, Classical Kinetic Theory of Fluids (John Wiley \& Sons, New York, 1977).

[16] J. W. Dufty and J. J. Brey, Math. Model. Nat. Phenom. 6, 19 (2011).

[17] A. J. Kovacs, Adv. Polym. Sci. (Fortschr. Hochpolym. Forsch.) 3, 394 (1963).

[18] L. Berthier and P. C. W. Holdsworth, Europhys. Lett. 58, 35 (2002).

[19] S. Mossa and F. Sciortino, Phys. Rev. Lett. 92, 045504 (2004).

[20] G. Aquino, A. Allahverdyan, and T. M. Nieuwenhuizen, Phys. Rev. Lett. 101, 015901 (2008).

[21] A. Prados and J. J. Brey, J. Stat. Mech. P02009 (2010).

[22] G. Diezemann and A. Heuer, Phys. Rev. E 83, 031505 (2011).

[23] A. Prados and E. Trizac, Phys. Rev. Lett. 112, 198001 (2014); E. Trizac and A. Prados, Phys. Rev. E 90, 012204 (2014).

[24] S. McNamara and J.-L. Barrat, Phys. Rev. E 55, 7767 (1997).
[25] J. S. Olafsen and J. S. Urbach, Phys. Rev. E 60, R2468 (1999).

[26] A. Puglisi, A. Gnoli, G. Gradenigo, A. Sarracino, and D. Villamaina, J. Chem. Phys. 136, 014704 (2012).

[27] J. J. Brey and D. Cubero, Phys. Rev. E 57, 2019 (1998).

[28] J. J. Brey, M. J. Ruiz-Montero, and F. Moreno, Phys. Rev. E 63, 061305 (2001).

[29] P. Melby et al., J. Phys.: Condens. Matter 17, S2689 (2005).

[30] A. Prevost, P. Melby, D. A. Egolf, and J. S. Urbach, Phys. Rev. E 70, 050301(R) (2004).

[31] J. S. Olafsen and J. S. Urbach, Phys. Rev. Lett. 81, 4369 (1998).

[32] M. G. Clerc, P. Cordero, J. Dunstan, K. Huff, N. Mújica, D. Risso, and G. Varas, Nat. Phys. 4, 249 (2008).

[33] M. P. Allen and D. J. Tildesley, Computer Simulations of Liquids (Clarendon Press, Oxford, 1987).

[34] T. Pöschel and T. Schwager, Computational Granular Dynamics (Springer, Berlin, 2005).

[35] E. Gradenigo, A. Sarracino, D. Villamaina, and A. Puglisi, Europhys. Lett. 96, 14004 (2011).

[36] V. Garzó and J. M. Montanero, Physica A 313, 336 (2002).

[37] P. Visco, A. Puglisi, A. Barrat, E. Trizac, and F. van Wijland, J. Stat. Phys. 125, 533 (2006).

[38] M. I. García de Soria, P. Maynar, and E. Trizac, Mol. Phys. 107, 383 (2009).

[39] P. Maynar, M. I. García de Soria, and E. Trizac, Eur. Phys. J. Spec. Top. 179, 123 (2009).

[40] S. R. Dahl, C. M. Hrenya, V. Garzó, and J. W. Dufty, Phys. Rev. E 66, 041301 (2002).

[41] V. Garzó, Europhys. Lett. 75, 521 (2006).

[42] N. V. Brilliantov and T. Pöschel, Europhys. Lett. 74, 424 (2006).

[43] J. M. Montanero and A. Santos, Granular Matter 2, 53 (2000). Although this paper deals with a three-dimensional system, the same is trivially expected for two dimensions.

[44] M. I. García de Soria, P. Maynar, and E. Trizac, Phys. Rev. E 85, 051301 (2012).

[45] G. Bird, Molecular Gas Dynamics and the Direct Simulation of Gas Flows (Clarendon, Oxford, 1994).

[46] A. García, Numerical Methods for Physics (Prentice Hall, Englewood Cliffs, NJ, 2000).

[47] J. J. Brey, M. J. Ruiz-Montero, and D. Cubero, Phys. Rev. E 54, 3664 (1996).

[48] M. I. García de Soria, P. Maynar, and E. Trizac, Phys. Rev. E 87, 022201 (2013). 